\title{
Early Prophylactic Hypothermia for Patients With Severe Traumatic Injury: Premature to Close the Case
}

\author{
Shan Min Chin and Didier Wion* \\ INSERM UMR1205, Faculté Médecine Pharmacie, Université Grenoble Alpes, La Tronche, France
}

Keywords: therapeutic hypothermia, traumatic brain injury, focal hypothermia, pro-resolving therapies, specialized proresolving mediators

\section{BACKGROUND}

In a recent well-designed and executed clinical trial, Cooper and collaborators found no evidence to support the use of early prophylactic hypothermia in patients with severe traumatic brain injury (TBI) (1). This study demonstrates that there is no role for the initiation of hypothermia during the acute phase of TBI $(1,2)$. However, it would be damaging to abandon the concept prematurely.

\section{INFLAMMATION ALSO PAVES THE WAY TO TISSUE REPAIR}

OPEN ACCESS

Edited by:

Peter Bergold,

SUNY Downstate Medical Center,

United States

Reviewed by:

W. Dalton Dietrich,

University of Miami, United States

Holly Elaine Hinson,

Oregon Health \& Science University,

United States

*Correspondence:

Didier Wion

didier.wion@univ-grenoble-alpes.fr

Specialty section:

This article was submitted to

Neurotrauma,

a section of the journal

Frontiers in Neurology

Received: 09 January 2019 Accepted: 20 March 2019

Published: 09 April 2019

Citation:

Chin SM and Wion D (2019) Early

Prophylactic Hypothermia for Patients With Severe Traumatic Injury:

Premature to Close the Case.

Front. Neurol. 10:344.

doi: 10.3389/fneur.2019.00344
As soon as trauma occurs, the inflammatory cascade begins to take place. The deleterious role of inflammation in the secondary injury response is well-documented, hence the rationale to attempt early prophylactic hypothermia in TBI. However, inflammation also initiates tissue repair and regeneration (3-6). We now know that the secondary injury response accompanies the regenerating and healing response. Rather than considering the injury cascades and resolving phases as two sequential antagonistic processes, we now understand them as two overlapping aspects of the same dynamic process dedicated to regeneration and healing (3-5). The resolving phase is an active process intrinsic to the progression of the inflammatory response. It is triggered by several pro-inflammatory mediators such as IL- $1 \beta$ and TNF- $\alpha$, that recruit, among others, IL-10, TGF $\beta$, and the members of a novel superfamily of lipid mediators called specialized pro-resolving mediators (SPMs) (3-6). The members of the SPMs family that include lipoxins, resolvins (Rvs), maresins, and protectins are central to this process $(3,7)$. They actively terminate inflammation and promote tissue regeneration $(3,7,8)$. Indeed, the temporal switch from the generation of proinflammatory mediators to the synthesis of SPMs is essential to the resolution of inflammation and healing $(3,7,8)$. Several SMPs have already been detected in human brain (9). As regards TBI, at least two members of the SPM family, namely Resolvin D1 and Protectin D1are identified in mouse brain tissue following stroke-injury (10). Recent experiments performed on rat also show that Resolvin D1 decreases remote neuro-inflammation and improves functional recovery after focal brain damage (11). Resolvin D2 also protects against cerebral ischemia/reperfusion injury (12).

Because the synthesis of SPMs is intrinsic to the inflammatory response, any uncontrolled braking of the inflammatory process can impair their synthesis, that would in turn affect the resolving phase and brain healing $(3,5-7)$. This could explain the paradoxical "resolution-toxic" side effects that some anti-inflammatory drugs or therapies may have $(3,5,13)$. In this emerging framework, where the inflammatory response is both a target and a tool for therapy $(3,5,7,14)$, many outstanding questions remain. For examples, how does prophylactic hypothermia affect the complex network of proinflammatory and proresolving mediators and thus the resolving phase of inflammation? More generally, to what extent does hypothermia delay the synthesis of SPMs and of other resolving factors or neuroprotective cytokines and impairs their function? Does the process of cooling and rewarming hinder the prophylactic potential of hypothermia by desynchronizing 
the resolving phase? What happens to the clearance of apoptotic cells and cell debris during and after hypothermia? Currently, we are still missing the answers to those questions. It would be rather surprising if hypothermia did not impair or delay some processes involved in tissue repair and regeneration. Combining a pro-resolving therapeutic strategy may be the necessary step to reveal the prophylactic potential of hypothermia in TBI.

\section{FOCAL HYPOTHERMIA: THE ALTERNATIVE TO WHOLE-BODY COOLING PROTOCOLS}

Another concern is the whole-body cooling protocol used in clinical trials. The potential complications of systemic hypothermia are well-known. They mainly include infection like pneumonia, and hypotension. Moreover, as regards the use of therapeutic hypothermia in TBI, a single optimal hypothermic temperature for all regions of the brain subjected to trauma probably does not exist. The effective prophylactic temperature is unlikely to be the same for the lesion core, the penumbra, and the remainder of the body. Importantly, these different optimal temperatures, once defined, will need to be finely tuned over time. We must handle hypothermia as a dynamic and local parameter. The alternative to whole-body cooling is focal hypothermia. It would allow deliverance of specific temperature gradients to local space-time frameworks without the side effects observed with whole-body hypothermia. The concept of focal brain hypothermia is not new. As early as 1940, T Fay tried to treat glioblastoma patients by implanting in the resection cavity metal capsules connected to refrigerated liquids (15). Nowadays, implantable focal cooling devices are in development to prevent epileptic seizures $(16,17)$ and glioma recurrence $(18)$. In a model of experimental cerebral ischemia, neuroprotective effects are obtained in free-moving rats with an implantable focal brain cooling device (19). In non-human primate an epidural cooling to $15^{\circ} \mathrm{C}$ with a cooling pad achieves a brain temperature to $34-35^{\circ} \mathrm{C}$ at $15 \mathrm{~mm}$ deep and $28-32^{\circ} \mathrm{C}$ at $10 \mathrm{~mm}$ deep (20). The same brain surface temperature of about $15^{\circ} \mathrm{C}$ was also recently achieved in non-human primates with a chronically implanted titanium cooling plate (21). These preclinical studies point to an important limitation of our current focal surface devices. The diffusion of hypothermia in the brain parenchyma is limited to around $15 \mathrm{~mm}$ due to thermal buffering by blood circulation (22), and to the low thermal conductivity of the brain (23). Hence, epidural focal cooling should benefit only patients with superficial neocortical lesions. For deeper focal traumatic lesions, and as long as we will not have any method to increase the brain cooling diffusion or mini invasive implantable intracerebral cooling devices (17), selective endovascular intracarotid infusion of cold saline remains the alternative to whole body cooling (24).

\section{REFERENCES}

1. Cooper DJ, Nichol AD, Bailey M, Bernard S, Cameron PA, Pili-Floury S, et al. Effect of early sustained prophylactic hypothermia on neurologic outcomes
Clearly, there are still many research and technical challenges ahead (25). It is in response to these challenges that we need to develop implantable microdevices capable to continuously and locally adjusting the optimal gradients of hypothermia in conjunction with pro-resolving therapies.

\section{CONCLUDING REMARKS}

The results of the trial conducted by Cooper et al showed that early hypothermia did not improve long-term favorable functional outcomes in patients with severe TBI. No effect of hypothermia on intracranial pressure is also found (1). However, it is premature to close the case of therapeutic hypothermia in TBI. Understanding the discrepancy between these results and the huge amount of convincing experimental evidence is now the clinical challenge we must overcome. Experimental evidence demonstrates that hypothermia can protect brain function by moderating immune inflammatory responses, reducing brain metabolism and edema. TBI is a highly heterogeneous condition, and not all the type of brain lesions would respond similarly to a standardized protocol of whole body cooling. In a certain way, we could say that the concept of therapeutic hypothermia is valid but that we do not know yet how to make it work in large randomized clinical trials. Patient stratification and the combination of therapeutic hypothermia with minimally invasive surgery and adjuvant therapies is probably necessary to reveal the therapeutic effects of hypothermia in RCTs. As regards the selfreinforcing cycle between inflammation and edema/intracranial pressure (26), we know that the anti-inflammatory effects of mild hypothermia combined with minimally invasive surgery can reduce brain edema $(27,28)$. Posttraumatic hypothermia also reduces the levels of MMP9 that is a major contributor to blood brain barrier disruption and edema $(29,30)$. Hence, therapeutic hypothermia remains a reasonable option in a multimodal approach as a rescue therapy for patients with poorly controlled intracranial pressure and/or cerebral edema. Another point to consider is the possibility to use therapeutic hypothermia to extend the therapeutic time window of some drugs (31).

There is a future for prophylactic hypothermia in TBI. It resides in patients stratification, and in the development of synergies between focal intelligent cooling devices, pro-resolving therapies $(32,33)$, and novel neuroprotective drugs (34).

\section{AUTHOR CONTRIBUTIONS}

All authors listed have made a substantial, direct and intellectual contribution to the work, and approved it for publication.

\section{FUNDING}

The research is funded by INSERM.

among patients with severe traumatic brain injury: the POLAR Randomized Clinical Trial. JAMA. (2018) 320:2211-20. doi: 10.1001/jama.2018.17075

2. Docherty A, Emelifeonwu J, Andrews PJD. Hypothermia after traumatic brain injury. JAMA. (2018) 320:2204-6. doi: 10.1001/jama.2018.17121 
3. Buckley CD, Gilroy DW, Serhan CN. Proresolving lipid mediators and mechanisms in the resolution of acute inflammation. Immunity. (2014) 40:315-27. doi: 10.1016/j.immuni.2014.02.009

4. Burda JE, Sofroniew MV. Reactive gliosis and the multicellular response to CNS damage and disease. Neuron. (2014) 81:229-48. doi: 10.1016/j.neuron.2013.12.034

5. Chabrol T, Berger F, Wion D. Inflammation and inflammatory diseases: how our language influences our therapeutic paradigms. Bioessays. (2018) 40:e1800103. doi: 10.1002/bies.201800103

6. Karin M, Clevers H. Reparative inflammation takes charge of tissue regeneration. Nature. (2016) 529:307-15. doi: 10.1038/nature17039

7. Serhan CN. Pro-resolving lipid mediators are leads for resolution physiology. Nature. (2014) 510:92-101. doi: 10.1038/nature13479

8. Serhan CN, Levy BD. Resolvins in inflammation: emergence of the pro-resolving superfamily of mediators. J Clin Invest. (2018) 128:2657-69. doi: 10.1172/JCI97943

9. Zhu M, Wang X, Hjorth E, Colas RA, Schroeder L, Granholm A-C, et al. Pro-resolving lipid mediators improve neuronal survival and increase A $\beta 42$ phagocytosis. Mol Neurobiol. (2016) 53:2733-49. doi: 10.1007/s12035-015-9544-0

10. Hong S, Lu Y, Yang R, Gotlinger KH, Petasis NA, Serhan CN. Resolvin D1, protectin D1, and related docosahexaenoic acid-derived products: analysis via electrospray/low energy tandem mass spectrometry based on spectra and fragmentation mechanisms. J Am Soc Mass Spectrom. (2007) 18:128-44. doi: 10.1016/j.jasms.2006.09.002

11. Bisicchia E, Sasso V, Catanzaro G, Leuti A, Besharat ZM, Chiacchiarini $\mathrm{M}$, et al. Resolvin D1 Halts remote neuroinflammation and improves functional recovery after focal brain damage Via ALX/FPR2 receptor-regulated MicroRNAs. Mol Neurobiol. (2018) 55:6894-905. doi: 10.1007/s12035-018-0889-Z

12. Zuo G, Zhang D, Mu R, Shen H, Li X, Wang Z, et al. Resolvin D2 protects against cerebral ischemia/reperfusion injury in rats. Mol Brain. (2018) 11:9. doi: 10.1186/s13041-018-0351-1

13. Gilroy DW, Colville-Nash PR, Willis D, Chivers J, Paul-Clark MJ, Willoughby DA. Inducible cyclooxygenase may have anti-inflammatory properties. Nat Med. (1999) 5:698-701. doi: 10.1038/9550

14. Lambertsen KL, Finsen B, Clausen BH. Post-stroke inflammation-target or tool for therapy? Acta Neuropathol. (in press). doi: 10.1007/s00401-018-1930-z

15. Fay T. Early experiences with local and generalized refrigeration of the human brain. J. Neurosurg. (1959) 16:239-59; discussion 259-60. doi: $10.3171 /$ jns.1959.16.3.0239

16. Hata K, Fujiwara K, Inoue T, Abe T, Kubo T, Yamakawa T, et al. Epileptic seizure suppression by focal brain cooling with recirculating coolant cooling system: modeling and simulation. IEEE Trans Neural Syst Rehabil Eng. (2019) 27:162-71. doi: 10.1109/TNSRE.2019.2891090

17. Smyth MD, Rothman SM. Focal cooling devices for the surgical treatment of epilepsy. Neurosurg Clin N Am. (2011) 22:533-46, vii. doi: 10.1016/j.nec.2011.07.011

18. Wion D. Therapeutic dormancy to delay postsurgical glioma recurrence: the past, present and promise of focal hypothermia. J Neurooncol. (2017) 133:447-54. doi: 10.1007/s11060-017-2471-3

19. Tauchi M, Tejada de Rink MM, Fujioka H, Okayama S, Nakamura K-I, Dietel B, et al. Targeted temperature management: peltier's element-based focal brain cooling protects penumbra neurons from progressive damage in experimental cerebral ischemia. Ther Hypothermia Temp Manag. (2018). 8:1-9. doi: 10.1089/ther.2017.0055

20. King C, Robinson T, Dixon CE, Rao GR, Larnard D, Nemoto CEM. Brain temperature profiles during epidural cooling with the ChillerPad in a monkey model of traumatic brain injury. J Neurotrauma. (2010) 27:1895-903. doi: $10.1089 /$ neu.2009.1178
21. Inoue $\mathrm{T}$, Fujii $\mathrm{M}$, Kida $\mathrm{H}$, Yamakawa $\mathrm{T}$, Maruta $\mathrm{Y}$, Tokiwa $\mathrm{T}$, et al. Epidural focal brain cooling abolishes neocortical seizures in cats and nonhuman primates. Neurosci Res. (2017) 122:35-44. doi: 10.1016/j.neures.2017. 04.007

22. Smyth MD, Han RH, Yarbrough CK, Patterson EE, Yang X-F, Miller JW, et al. Temperatures achieved in human and canine neocortex during intraoperative passive or active focal cooling. Ther Hypothermia Temp Manag. (2015) 5:95103. doi: 10.1089/ther.2014.0025

23. Osorio I, Chang F-C, Gopalsami N. Seizure control with thermal energy? Modeling of heat diffusivity in brain tissue and computer-based design of a prototype mini-cooler. Epilepsy Behav. (2009) 16:203-11. doi: 10.1016/j.yebeh.2009.08.014

24. Choi JH, Marshall RS, Neimark MA, Konstas AA, Lin E, Chiang YT, et al. Selective brain cooling with endovascular intracarotid infusion of cold saline: a pilot feasibility study. AJNR Am J Neuroradiol. (2010) 31:928-34. doi: 10.3174/ajnr.A1961

25. Choi JH, Pile-Spellman J. Selective brain hypothermia. Handb Clin Neurol. (2018) 157:839-52. doi: 10.1016/B978-0-444-64074-1.00052-5

26. Shlosberg D, Benifla M, Kaufer D, Friedman A. Blood-brain barrier breakdown as a therapeutic target in traumatic brain injury. Nat Rev Neurol. (2010) 6:393-403. doi: 10.1038/nrneurol.2010.74

27. Staykov D, Schwab S, Dörfler A, Kollmar R. Hypothermia reduces perihemorrhagic edema after intracerebral hemorrhage: but does it influence functional outcome and mortality? Ther Hypothermia Temp Manag. (2011) 1:105-6. doi: 10.1089/ther.2011.0004

28. Zhao J, Mao Q, Qian Z, Zhu J, Qu Z, Wang C. Effect of mild hypothermia on expression of inflammatory factors in surrounding tissue after minimally invasive hematoma evacuation in the treatment of hypertensive intracerebral hemorrhage. Exp Ther Med. (2018) 15:4906-10. doi: 10.3892/etm. 2018.6014

29. Jia F, Pan Y, Mao Q, Liang Y, Jiang J. Matrix metalloproteinase-9 expression and protein levels after fluid percussion injury in rats: the effect of injury severity and brain temperature. J Neurotrauma. (2010) 27:1059-68. doi: $10.1089 /$ neu.2009.1067

30. Truettner JS, Alonso OF, Dietrich WD. Influence of therapeutic hypothermia on matrix metalloproteinase activity after traumatic brain injury in rats. $J$ Cereb Blood Flow Metab. (2005) 25:1505-16. doi: 10.1038/sj.jcbfm.9600150

31. Mohamadpour M, Whitney K, Bergold PJ. The importance of therapeutic time window in the treatment of traumatic brain injury. Front Neurosci. (2019) 13:7. doi: 10.3389/fnins.2019.00007

32. Fullerton JN, Gilroy DW. Resolution of inflammation: a new therapeutic frontier. Nat Rev Drug Discov. (2016) 15:551-67. doi: 10.1038/nrd.2016.39

33. Yin P, Wei Y, Wang X, Zhu M, Feng J. Roles of specialized pro-resolving lipid mediators in cerebral ischemia reperfusion injury. Front Neurol. (2018) 9:617. doi: 10.3389/fneur.2018.00617

34. Gao X-Y, Huang J-O, Hu Y-F, Gu Y, Zhu S-Z, Huang K-B, et al. Combination of mild hypothermia with neuroprotectants has greater neuroprotective effects during oxygen-glucose deprivation and reoxygenation-mediated neuronal injury. Sci Rep. (2014) 4:7091. doi: 10.1038/srep07091

Conflict of Interest Statement: The authors declare that the research was conducted in the absence of any commercial or financial relationships that could be construed as a potential conflict of interest.

Copyright $@ 2019$ Chin and Wion. This is an open-access article distributed under the terms of the Creative Commons Attribution License (CC BY). The use, distribution or reproduction in other forums is permitted, provided the original author(s) and the copyright owner(s) are credited and that the original publication in this journal is cited, in accordance with accepted academic practice. No use, distribution or reproduction is permitted which does not comply with these terms. 